\title{
Doença de Kawasaki: experiência clínica em hospital universitário
}

\author{
Kawasaki disease: clinical experience in a university hospital
}

\section{Angela Esposito Ferronato ${ }^{1}$, Selma Betta Ragazzi², Maki Hirose ${ }^{1}$, Débora Morais Cardoso ${ }^{1}$, Alfredo Elias Gilio ${ }^{3}$}

\section{RESUMO}

Objetivo: A doença de Kawasaki é uma vasculite sistêmica aguda de etiologia desconhecida. Seu diagnóstico baseia-se em critérios clínicos. O objetivo deste estudo foi descrever os casos de pacientes com doença de Kawasaki internados no Hospital Universitário da Universidade de São Paulo entre janeiro/2000 e junho/2008.

Métodos: Dentre todos os pacientes internados na Enfermaria de Pediatria no período acima, foram selecionados aqueles cujo CID de alta foi doença de Kawasaki. Realizou-se estudo descritivo por meio da análise dos prontuários dessas crianças.

Resultados: Foram encontrados 18 casos. A média de internações foi de 2,1 casos/ano. A idade variou de três meses a nove anos. A proporção meninos:meninas foi 1:1,25. Receberam outros diagnósticos prévios 17 pacientes, sendo escarlatina em 2/3 dos casos. O tempo de febre antes do diagnóstico variou de cinco a 11 dias. Nove crianças apresentaram quatro sinais sugestivos de doença de Kawasaki; oito apresentaram cinco sinais e uma apresentou dois sinais, o que foi considerado doença de Kawasaki incompleta. Receberam gamaglobulina 15 crianças (entre o sexto e o décimo dias de evolução) e 11 (73\%) ficaram afebris após infusão da medicação. Os demais tiveram febre até 24 horas após a administração. Todos os pacientes realizaram ecocardiograma e três apresentaram aneurisma leve da coronária.

Conclusões: A doença de Kawasaki é habitualmente confundida com outras doenças, o que causa retardo no tratamento e aumento no risco de complicações cardíacas.

Palavras-chave: doença de Kawasaki; síndrome do linfonodo mucocutâneo; vasculite.

\section{ABSTRACT}

Objective: Kawasaki disease is an acute systemic vasculitis of unknown etiology. Its diagnosis is based on clinical criteria. This study aimed to describe Kawasaki disease cases treated at the University Hospital of Universidade de São Paulo, from January/2000 to June/2008.

Methods: Among all patients admitted to the pediatric ward during this period, patients whose discharge ICD was Kawasaki disease were selected. A descriptive study was carried out by analyzing the records of these children.

Results: 18 cases were found, with an average of 2.1 cases/ year. Patients varied from three to nine years old. The boys/ girls ratio was $1 / 1.25$. Seventeen patients had previously been misdiagnosed with other diseases, being 2/3 of them scarlet fever. Prior to diagnosis, fever had persisted for five to 11 days. Nine patients showed four suggestive signs of Kawasaki disease, eight patients showed five signs, and one patient had two suggestive signs, which was considered as incomplete Kawasaki disease. Gammaglobulin was administered to 15 children (between the $6^{\text {th }}-10^{\text {th }}$ day of the disease), with 11 (73\%) of them having no fever following the administration. The other ones had fever for up to 24 hours following gammaglobolin use. All patients were evaluated by echocardiograms, and three of them showed mild coronary aneurysm.

Conclusions: Kawasaki disease is usually misdiagnosed, thereby delaying treatment and increasing the risk of heart.

Key-words: Kawasaki disease; mucocutaneous lymph node syndrome; vasculitis.
Instituição: Hospital Universitário da Universidade de São Paulo (USP), São Paulo, SP, Brasil

${ }^{1}$ Médica Assistente da Enfermaria de Pediatria do Hospital Universitário da USP, São Paulo, SP, Brasil

2Mestre; Médica Chefe da Enfermaria de Pediatria do Hospital Universitário da USP, São Paulo, SP, Brasil

${ }^{3}$ Doutor; Diretor da Divisão de Pediatria do Hospital Universitário da USP, São Paulo, SP, Brasil
Endereço para correspondência:

Angela Esposito Ferronato

Avenida Professor Lineu Prestes, 2.565 - Cidade Universitária CEP 05508-000 - São Paulo/SP

E-mail: angela@hu.usp.br

Conflitos de interesse: nada a declarar

Recebido em: 22/6/09

Aprovado em: 30/11/09 


\section{Introdução}

A doença de Kawasaki (DK) é uma vasculite sistêmica aguda, de origem desconhecida, que ocorre predominantemente em lactentes e crianças menores de cinco anos de idade ${ }^{(1-4)}$. A primeira descrição da doença ocorreu em 1967, no Japão, por Tomisaku Kawasaki. Sua morbidade está relacionada às alterações cardíacas, que podem aparecer tardiamente ${ }^{(5-7)}$, sendo mais frequentes os aneurismas de coronárias e as ectasias de vasos sanguíneos cardíacos que acometem de 15 a $25 \%$ das crianças não tratadas ${ }^{(1,2,8)}$. Nos Estados Unidos, a DK tem superado a febre reumática como causa de doença cardíaca adquirida na infância ${ }^{(3,8,9)}$.

A etiologia da DK é desconhecida, porém algumas características clínicas e epidemiológicas sugerem fortemente uma causa infecciosa desencadeante ${ }^{(1,2,10)}$. A infecção levaria à formação de superantígenos que deflagrariam respostas imunológicas em pacientes geneticamente predispostos.

Não há exame subsidiário específico para o diagnóstico da DK, que se baseia na presença de febre por cinco dias ou mais e quatro dos seguintes sinais: exantema, conjuntivite não-exsudativa, alterações de mucosa (eritema, fissuras, língua em framboesa), adenomegalia não-supurativa e alterações de extremidades (eritema, edema e descamação) ${ }^{(1-3)}$. Os sinais clínicos não são concomitantes, sendo comum o diagnóstico inicial de outras doenças, como escarlatina, exantema viral ou quadros alérgicos. Mesmo com o preenchimento completo dos critérios, é frequente a demora no diagnóstico correto de DK e o atraso no início do tratamento adequado, favorecendo o aparecimento das complicações ${ }^{(7,11,12) .}$ Além disso, existem as formas incompletas ou atípicas, que também podem evoluir com acometimento coronariano ${ }^{(13-16)}$.

O diagnóstico precoce da doença é importante, pois o tratamento na fase aguda reduz o processo inflamatório nas artérias coronarianas e previne a trombose ${ }^{(17-19)}$. O tratamento consiste no uso de gamaglobulina $(2 \mathrm{~g} / \mathrm{kg})$ em dose única, e associado à aspirina de $80 \mathrm{a} 100 \mathrm{mg} / \mathrm{kg} / \mathrm{dia}$ em doses fracionadas a cada seis horas. Essa dose de aspirina deve ser mantida por duas semanas ou até 48 a 72 horas após o término da febre, sendo posteriormente reduzida para 3 a $5 \mathrm{mg} / \mathrm{kg}$ uma vez ao dia, até não haver evidência de alteração coronariana, ou até seis a oito semanas após início da doença ${ }^{(17)}$. O acompanhamento adequado do paciente com aneurisma previne a isquemia miocárdica e o infarto do miocárdio ${ }^{(1-3,8)}$.

O objetivo deste trabalho foi descrever as características epidemiológicas, clínicas e laboratoriais dos casos de DK internados no Hospital Universitário da Universidade de São Paulo, entre janeiro de 2000 e junho de 2008.

\section{Métodos}

O Hospital Universitário é regionalizado e presta atendimento de nível secundário à população da região do Butantã - bairro da cidade de São Paulo (SP), aos funcionários e aos dependentes de funcionários da universidade. Na Enfermaria Geral de Pediatria, são internadas crianças com idade entre 29 dias a 15 anos incompletos, com um total de aproximadamente 1.400 internações por ano. O hospital dispõe de um sistema informatizado, por meio do qual todos os diagnósticos de internação, evolução e alta são catalogados de acordo com a Classificação Internacional de Doenças (CID) e ficam à disposição para pesquisa. Durante a internação, todos os casos clínicos são analisados e discutidos com a equipe de residentes e assistentes, havendo sempre consenso quanto ao diagnóstico final. Para realizar este estudo, foi solicitado à informática um levantamento de todos os casos internados no período de janeiro de 2000 a junho de 2008 cujo diagnóstico de alta tenha sido de DK.

Os dados foram extraídos dos prontuários por meio do preenchimento de protocolo clínico que continha as seguintes variáveis: idade; sexo; raça; mês e ano da internação; tempo de internação; diagnósticos e tratamentos prévios; tempo de febre e a temperatura máxima; presença ou ausência de exantema, alterações de extremidade (edema/descamação), enantema, conjuntivite e linfonodos cervicais. Foram revistos os exames laboratoriais relacionados à DK: contagem de leucócitos e plaquetas no hemograma, marcadores inflamatórios (velocidade de hemossedimentação e proteína C-reativa), alanina transaminase (TGP), aspartato transaminase (TGO), bem como radiografia simples de tórax, eletrocardiograma e ecocardiograma.

Analisaram-se também os dados relacionados ao tratamento e à evolução dos casos, como administração da gamaglobulina, ácido acetilsalicílico (AAS), doses, tempo de doença em que as medicações foram introduzidas e tempo para resolução da febre.

\section{Resultados}

No período de janeiro de 2000 a junho de 2008, foram internadas 18 crianças com diagnóstico de DK à admissão ou na evolução (média de 2,1 casos ao ano). A idade variou 
de três meses a nove anos e dois meses, com mediana de três anos e dois meses. Metade dos pacientes apresentava idade entre um ano e seis meses e cinco anos; oito crianças eram do sexo feminino e dez do masculino, com uma proporção de 1:1,25 entre meninas e meninos. Não foi possível analisar a sazonalidade da doença em neste Serviço devido ao pequeno número de casos encontrados.

A Tabela 1 mostra os dados relativos aos pacientes avaliados e suas características clínicas. Com exceção de um, todos os pacientes tiveram diagnósticos de outras doenças antes de DK. Em muitos casos, havia mais de um diagnóstico anterior (Tabela 2). Escarlatina foi o diagnóstico prévio realizado com maior frequência. Devido a estes diagnósticos estabelecidos anteriormente, várias crianças receberam antimicrobiano ou outros medicamentos no momento da internação. Amoxicilina ou penicilina benzatina foram os mais prescritos, devido ao diagnóstico de amigdalite ou escarlatina (oito crianças), seguidos de cefalotina ou oxacilina (duas crianças), cefuroxime e ceftriaxone (uma criança).

A duração da febre variou de cinco a 11 dias, com média de 8,4 dias. A temperatura axilar máxima variou entre 38,4 e $40^{\circ} \mathrm{C}$, com média de $39,0^{\circ} \mathrm{C}$. Além da febre por mais de cinco dias, 17 pacientes apresentaram pelo menos quatro dos cinco critérios da doença. As alterações clínicas descritas com maior frequência foram: exantema e conjuntivite em 16 pacientes, seguida de alteração de extremidades, em 15. Enantema e linfonodos cervicais foram descritos em 12 pacientes.

Um dos pacientes descritos apresentava menos de quatro critérios clínicos e foi considerado DK incompleto. Esse paciente, no momento do diagnóstico, tinha um ano e cinco meses de idade, era do sexo feminino e mostrava somente enantema e alterações de extremidades (edema e descamação). Nessa criança, chamava atenção, nos exames subsidiários, a presença de leucocitúria, a elevação de proteína C-reativa e a meningite asséptica. A hipótese diagnóstica de DK foi feita somente após nove dias de febre e a criança ficou afebril logo após a administração da gamaglobulina. A presença de plaquetose no hemograma (644.000 plaquetas $/ \mathrm{mm}^{3}$ ) uma semana após o início da doença e um aneurisma coronoariano, encontrado no segundo ecocardiograma realizado na oitava semana da doença (o qual estava normal na segunda semana de doença), reforçaram posteriormente o diagnóstico.

As demais alterações clínicas encontradas nesses 18 pacientes foram irritabilidade (quatro casos), artralgia (dois), diarreia (dois), hepatomegalia (dois), sinais de irritação meníngea (dois) e hematúria macroscópica (um).
A coleta de exames laboratoriais não foi homogênea para todos os casos. A Tabela 3 mostra as alterações encontradas. A proteína C-reativa elevada foi observada em mais de $70 \%$ dos pacientes, seguida pela leucocitúria em 64\% e leucocitose no hemograma (44\%). O eletrocardiograma foi realizado em cinco pacientes e nenhum exame apresentou alterações significantes. A taquicardia sinusal foi a anormalidade mais observada. Doze pacientes realizaram raio $\mathrm{X}$ de tórax e somente em um havia descrição de algum grau de congestão e cardiomegalia moderada. O exame de liquor foi realizado em quatro dos 18 pacientes: dois com resultados normais e dois com aumento de celularidade (15 e 19 células, respectivamente; ambos com predomínio de linfócitos e monócitos).

A introdução da gamaglobulina ocorreu entre o sexto e o décimo dia de doença. Três dos 18 casos não receberam gamaglobulina. Dos pacientes que não receberam gamaglobulina, dois ficaram afebris no quinto dia de doença e um teve o diagnóstico de DK no oitavo dia de doença,ficando sem febre no $11^{\circ}$ dia (idade de cinco anos e quatro meses). Dos 15 pacientes que receberam gamaglobulina, 11 (73\%) não tiveram nenhum pico febril após a infusão da mesma. Quatro tiveram pelo menos um pico de febre até 24 horas após e permaneceram afebris desde então. Nenhum paciente apresentou febre após 24 horas da infusão da gamaglobulina.

Dos 18 pacientes, 17 receberam AAS em alta dose (80 a $100 \mathrm{mg} / \mathrm{kg} / \mathrm{dia}$ ), sendo que o período de uso dessa medicação variou entre dois e 12 dias. Todos os 18 pacientes realizaram ecocardiograma. O primeiro exame ocorreu entre a primeira e a segunda semana de evolução. Nesse período, dois pacientes apresentavam alteração ecocardiográfica. Um deles teve discreto aumento da coronária esquerda, que regrediu posteriormente (exame repetido na quarta e oitava semanas, ambos normais). $\mathrm{O}$ outro paciente evidenciava moderado comprometimento difuso do miocárdio, o qual não foi observado em novo exame realizado na mesma semana. $\mathrm{O}$ exame da quinta semana de evolução dessa criança mostrou pequena dilatação do óstio da coronária esquerda (exame no limite da normalidade). O ecocardiograma foi repetido pelo menos mais uma vez em 13 pacientes, em período que variou entre a terceira e a $24^{a}$ semanas de evolução. Em apenas um dos pacientes, na oitava semana de evolução da doença, foi observado aneurisma pequeno de coronária. Um dos pacientes que apresentou aneurisma de coronária não havia recebido gamaglobulina, pois, no momento do diagnóstico, estava afebril e com mais de 11 dias de história de doença. 


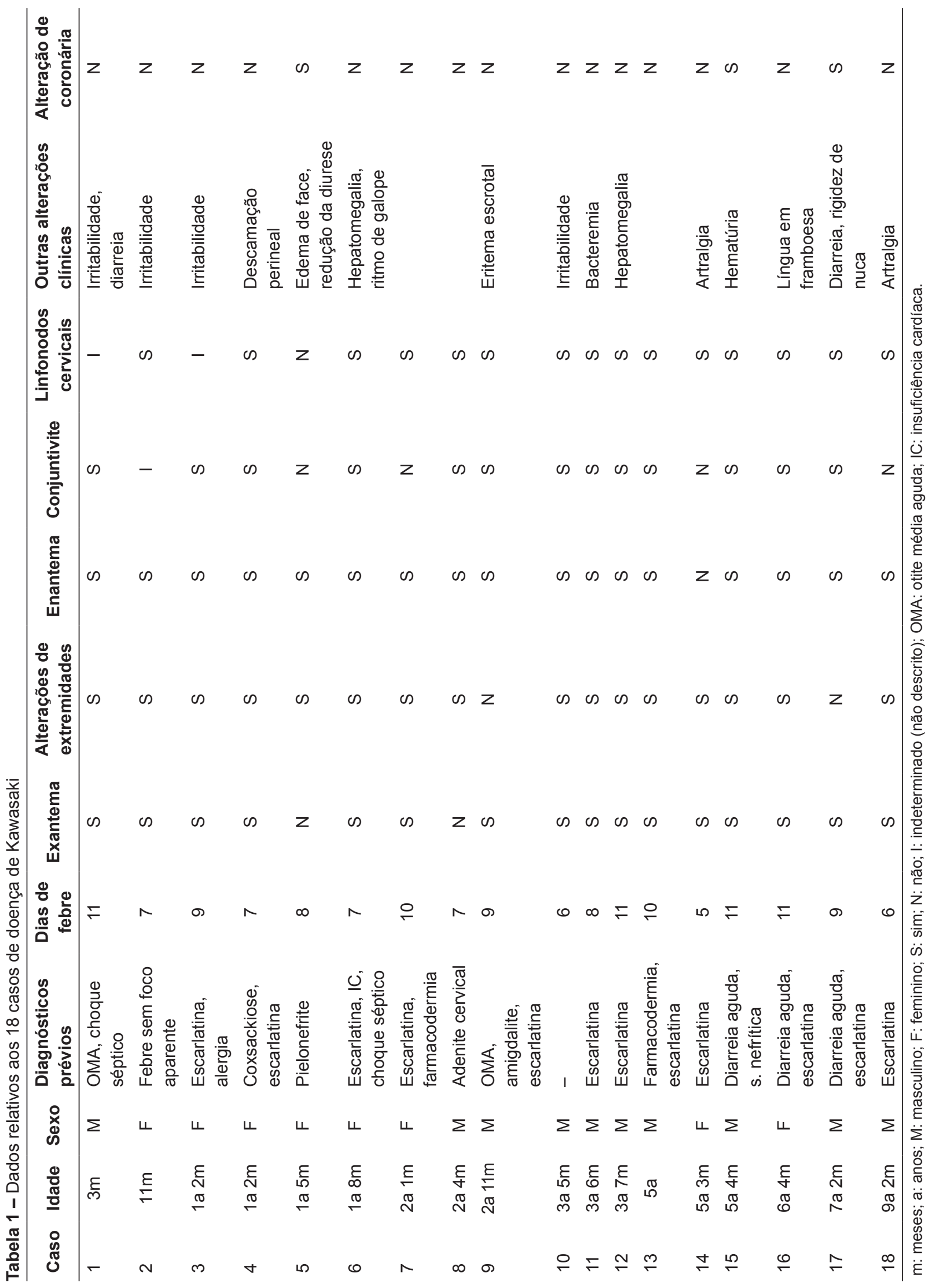




\section{Discussão}

A DK, descrita desde 1967, permanece uma doença com muitas características a serem observadas ${ }^{(5,11)}$. A falta de exames diagnósticos específicos e a variedade de sintomas que surgem no início da manifestação da doença podem levar, muitas vezes, a erro no diagnóstico inicial, o que retarda o diagnóstico e o tratamento específico ${ }^{(11,12)}$. Esse diagnóstico é mais difícil de ser estabelecido nos menores de um ano e nas crianças maiores de cinco anos ${ }^{(20,21)}$. Como verificado neste estudo, é muito frequente o diagnóstico inicial de outras doenças infecciosas ou alérgicas, sendo o diagnóstico prévio mais comum o da escarlatina, provavelmente pelas características do exantema e enantema ${ }^{(10,11)}$.

A febre geralmente é alta, com picos de $39^{\circ} \mathrm{Ce}$, em alguns casos, de $40^{\circ} \mathrm{C}$, podendo durar, na ausência de tratamento, cerca de dez dias ou até por três a quatro semanas, raramente mais do que isso. Com terapêutica adequada, a febre geralmente desaparece em dois dias ${ }^{(1-3)}$, o que ocorreu em todos os casos aqui descritos. Em $75 \%$ dos pacientes, não houve mais febre após a gamaglobulina e, em $25 \%$, a febre teve duração de até 24 horas após a infusão da gamaglobulina.

Alterações de extremidades são frequentes, surgindo eritema, edema de mãos e pés (frequentemente doloroso) e descamação, que se segue entre duas a três semanas. O exantema surge geralmente no quinto dia de febre e pode ter manifestações variáveis, como o aspecto macular, escarlatiniforme, urticariforme, multiforme-like ou eritrodermia e, raramente, papular. Muitas vezes, o aspecto do exantema leva o pediatra a fazer diagnóstico de exantema viral, escarlatina ou até mesmo de reação alérgica ${ }^{(2,3,11)}$. O exantema, uma das características clínicas mais descritas nos pacientes com $\mathrm{DK}^{(1-3)}$, é extenso, com envolvimento de tronco e extremidades, acentuando-se em região de períneo, com descamação precoce nesse local. No presente estudo, 16 pacientes (88\%) apresentaram exantema em algum momento da evolução da doença.

As alterações cardiovasculares na fase aguda podem ser proeminentes. Durante esse período, o pericárdio, o miocárdio, o endocárdio, as válvulas e artérias coronarianas podem ser comprometidos ${ }^{(2,8,22)}$. A ausculta cardíaca revela precórdio hiperdinâmico, taquicardia e ritmo de galope, com função miocárdica alterada, podendo simular choque séptico, miocardite ou outras causas de insuficiência cardíaca aguda $^{(22)}$. Um dos pacientes descritos apresentou quadro de insuficiência cardíaca aguda, simulando quadro séptico, o que retardou o diagnóstico de DK.

Os lábios e a cavidade oral são acometidos frequentemente com eritema e as características fissuras labiais, que, muitas vezes, chamam a atenção para o diagnóstico. A língua em framboesa pode ser um fator que contribui para o diagnóstico inicial de escarlatina, principalmente quando acompanhada de exantema escarlatiniforme $e^{(1,2,11)}$. O enantema foi descrito também em 12 pacientes (66\%) do presente estudo.

A adenomegalia cervical é a característica menos descrita na literatura, sendo na maioria das vezes unilateral. Mede cerca de $1,5 \mathrm{~cm}$ e pode ser confundida com adenite bacteriana $^{(1,2)}$. A adenomegalia cervical observada em 12 (66\%) dos 18 pacientes descritos.

Além dos critérios para diagnóstico de DK, outros achados clínicos e laboratoriais também são frequentes e podem auxiliar na suspeita do diagnóstico ou confundir com outras doenças. As alterações cardiovasculares mais descritas na fase aguda são: insuficiência cardíaca congestiva, miocardite, pericardite, regurgitação valvar, anormalidades de coronárias, aneurismas em outras artérias que não coronárias ${ }^{(1,2)}$.

Tabela 2 - Diagnósticos prévios à doença de Kawasaki

\begin{tabular}{lc}
\hline Diagnósticos prévios* & Número de casos \\
\hline Escarlatina & 12 \\
Farmacodermia /alergia/eritrodermia & 3 \\
Doença diarreica aguda & 3 \\
Amigdalite bacteriana & 2 \\
Otite média aguda & 2 \\
Choque séptico & 2 \\
Adenite bacteriana & 1 \\
Coxsackiose & 1 \\
Síndrome nefrítica & 1 \\
Pielonefrite & 1
\end{tabular}

*Vários pacientes tiveram mais de um diagnóstico prévio.

Tabela 3 - Resultados de exames laboratoriais e porcentagem de exames alterados

\begin{tabular}{lccc}
\hline & Ponto de corte & Número de pacientes & Exames alterados (\%) \\
\hline Proteína C-reativa elevada $(\mathrm{mg} / \mathrm{L})$ & $>50$ & 17 & 70,6 \\
Leucocitúria $\left(\mathrm{n} / \mathrm{mm}^{3}\right)$ & $>35.000$ & 17 & 64,7 \\
Leucocitose $\left(\mathrm{n} / \mathrm{mL}^{3}\right)$ & $>15.000$ & 18 & 44,4 \\
Alanina transaminase alterada $(\mathrm{mg} / \mathrm{dL})$ & $>60$ & 14 & 35,7 \\
Aspartato transaminase alterada $(\mathrm{mg} / \mathrm{dL})$ & $>60$ & 14 & 21,4 \\
Plaquetose $\left(\mathrm{n}^{\circ} / \mathrm{mm}^{3}\right)$ & $>500.000$ & 18 & 11,1 \\
\hline
\end{tabular}


Um dos pacientes da presente casuística teve diagnóstico inicial de choque séptico com falência cardíaca por apresentar sinais clínicos de insuficiência cardíaca congestiva com hepatomegalia e ausculta de ritmo de galope, retardando o diagnóstico de DK. Alterações articulares, como artrite e artralgias são frequentemente observadas e, muitas vezes, acompanham as demais alterações de extremidades ${ }^{(1-3)}$. O trato gastrintestinal também apresenta alterações que incluem diarreia, vômitos, dor abdominal e alterações hepáticas. No sistema nervoso central, é possível verificar irritabilidade, meningite asséptica e alterações auditivas ${ }^{(1,3,4)}$. A irritabilidade foi observada em quatro dos pacientes aqui relatados, com maior frequência nos lactentes. A piúria estéril é descrita em mais de $80 \%$ dos $\operatorname{casos}^{(1,2)}$. No levantamento do presente estudo, o aumento significativo de leucócitos na urina foi observado em cerca de $70 \%$ dos casos. Outros achados podem incluir eritema e enduração no local da vacina por BCG e uveíte anterior ${ }^{(1)}$.

As alterações laboratoriais mais observadas são hemograma com leucocitose e neutrofilia, além de aumento dos valores de exames de fase aguda, como velocidade de hemossedimentação e proteína C-reativa ${ }^{(1-5)}$. Neste levantamento, $70 \%$ dos pacientes apresentavam aumento significativo da proteína C-reativa. Elevação de transaminases também é frequente.

É importante lembrar as formas incompletas ou atípicas, nas quais os critérios clínicos não são todos preenchidos. $\mathrm{Na}$ descrição original da $\mathrm{DK}$, cinco dos 50 pacientes apresentavam a forma incompleta da doença ${ }^{(5)}$. Nesses casos, o diagnóstico é postergado pela ausência do número mínimo de características. A doença, muitas vezes, só é diagnosticada após se constatar o acometimento das coronárias ${ }^{(13-15)}$. Observou-se, na casuística desse estudo, apenas um paciente com DK incompleta, para quem o diagnóstico só foi estabelecido após o nono dia de doença. Um aneurisma de coronária foi detectado no segundo ecocardiograma, realizado na oitava semana de doença.

Nas crianças mais velhas, o risco de lesão coronariana é maior e pode estar relacionada ao diagnóstico mais tardio ${ }^{(8,21)}$. A eficácia da gamaglobulina administrada na fase aguda de DK para redução da prevalência da anormalidade de artérias coronarianas é bem estabelecida. O exato mecanismo de ação da mesma permanece desconhecido ${ }^{(1-3)}$. A terapêutica deve ser indicada nos primeiros dez dias de doença e, se possível, nos primeiros sete dias. Quanto mais precoce é o inicio da gamaglobulina, melhor será seu efeito em prevenir alterações coronarianas. O beneficio da utilização de gamaglobulina após o $14^{\circ}$ dia de doença é controverso ${ }^{(7,23)}$. Entretanto, qual- quer criança com DK que mantenha evidência de processo inflamatório, incluindo febre ou aumento de marcadores inflamatórios, com ou sem anormalidade coronária, deve ser tratada com gamaglobulina, mesmo quando o diagnóstico é feito após o décimo dia de doença ${ }^{(23)}$. Na casuística aqui relatada, identificou-se um caso em que o diagnóstico só foi feito após o $11^{\circ}$ dia de doença e já não apresentava sinais evidentes de processo inflamatório ativo. Estava afebril, com exames de marcadores inflamatórios normais. Esse paciente apresentou alteração leve de coronária no ecocardiograma de controle.

A DK pode recorrer em 3\% das crianças acometidas e, nessa situação, há maior risco de lesão $\operatorname{coronariana}^{(9)}$. No período de estudo, não se observou reinternação devido à recorrência da DK. Os pacientes sem alterações ecocardiográficas receberam alta do ambulatório após o terceiro exame normal e os que apresentaram qualquer variação no exame foram encaminhados a um serviço de cardiopediatria de referência.

Nos Estados Unidos, é típica a constatação de uma sazonalidade para a doença, sendo mais prevalente no inverno e início da primavera ${ }^{(24,25)}$. Não foi possível, neste estudo, constatar sazonalidade devido ao pequeno número de casos descritos. A DK é mais prevalente em meninos e $75 \%$ dos casos ocorrem em menores de cinco anos. A mortalidade no Japão é de 0,008\% e, nos Estados Unidos, a mortalidade hospitalar é de $0,17 \%$, em todos os casos secundária a sequelas cardíacas $^{(2-4)}$. O pico de morte ocorre entre 15 e 45 dias após o início da febre ${ }^{(2,7)}$. Não se observaram óbitos associados à DK no Hospital Universitário. No entanto, morte súbita por infarto agudo do miocárdio pode ocorrer anos depois. Casos de infarto agudo do miocárdio, fatais ou não, em adultos jovens, podem ser atribuídos a DK não-diagnosticada ${ }^{(2,7,8)}$.

No Brasil, não existem estudos sobre a prevalência da DK, mas, nos Estados Unidos, registra-se aumento de sua prevalência, de tal forma que a DK é atualmente a maior causa de cardiopatia adquirida na infância ${ }^{(11)}$. Em nosso meio, a principal causa de cardiopatia adquirida na infância ainda é a febre reumática ${ }^{(26)}$, porém há poucos dados a respeito de aumento da incidência de cardiopatias secundárias à DK.

Considerando que este estudo retrata um limitado número de casos da doença, não se pode afirmar que os dados encontrados retratam a realidade da doença no Brasil. Apesar disso, observa-se que algumas características da população aqui analisada são semelhantes às relatadas na literatura, como a idade, as manifestações clínicas, os exames laboratoriais e a resposta à gamaglobulina. Além disso, foi possível constatar 
ser bastante frequente o diagnóstico prévio de outras doenças infecciosas ou alérgicas, antes de se chegar à hipótese de DK. É importante que o pediatra tenha sempre em mente a DK como diagnóstico diferencial nas doenças febris para não retardar o seu diagnóstico e tratamento, o que leva ao aumento do risco de doenças coronarianas e sequelas cardíacas.

\section{Referências bibliográficas}

1. Burns JC, Glodè MP. Kawasaki syndrome. Lancet 2004;364:533-44.

2. Newburger JW, Takahashi M, Gerber MA, Gewitz MH, Tani LY, Burns JC et al. Diagnosis, treatment, and long-term management of Kawasaki disease: a statement for health professionals from the Committee on Rheumatic Fever, Endocarditis and Kawasaki Disease, Council on Cardiovascular Disease in the Young, American Heart Association. Pediatrics 2004;114:1708-33.

3. Pinna GS, Kafetzis DA, Tselkas OI, Skevaki CL. Kawasaki disease: an overview. Curr Opin Infect Dis 2008;21:263-70.

4. Newburger JW, Fulton DR. Kawasaki disease. Curr Opin Pediatr 2004;16: 508-14.

5. Burns JC. Commentary: translation of Dr. Tomisaku Kawasaki's original report of fifty patients in 1967. Pediatr Infect Dis J 2002;21:993-5.

6. Gedalia A. Kawasaki disease: 40 years after the original report. Curr Rheumatol Rep 2007;9:336-41.

7. Satou GM, Giamelli J, Gewitz MH. Kawasaki disease: diagnosis, management, and long-term implications. Cardiol Rev 2007;15:163-9.

8. Belay ED, Maddox RA, Holman RC, Curns AT, Ballah K, Schonberger LB. Kawasaki syndrome and risk factors for coronary artery abnormalities: United States, 1994-2003. Pediatr Infect Dis J 2006;25:245-9.

9. Newburger JW. Kawasaki disease: who is at risk? J Pediatr 2000;137: 149-52.

10. Vincent $P$, Salo E, Skurnik M, Fukushima H, Simonet M. Similarities of Kawasaki disease and Yersinia pseudotuberculosis infection epidemiology. Pediatr Infect Dis J 2007;26:629-31.

11. Burns JC. The riddle of Kawasaki disease. N England J Med 2007;356: 659-61.

12. Minich LL, Sleeper LA, Atz AM, McCrindle BW, Lu M, Colan SD et al. Delayed diagnosis of Kawasaki disease: what are the risk factors? Pediatrics 2007;120:e1434-40.

13. Sonobe T, Kiyosawa N, Tsuchiya K, Aso S, Imada Y, Imai Y et al. Prevalence of coronary artery abnormality in incomplete Kawasaki disease. Pediatr Int 2007;49:421-6.
14. Levy M, Koren G. Atypical Kawasaki disease: analysis of clinical presentation and diagnostic clues. Pediatr Infect Dis J 1990;9:122-6.

15. Rowley $\mathrm{AH}$. Incomplete (atypical) Kawasaki disease. Pediatr Infect Dis J 2002;21:563-5.

16. Tomikawa SO, Sakamoto RA, Gonçalves AM, Rodrigues Neto AJ, Sakane PT. A dificuldade diagnóstica na doença de Kawasaki: relato de caso. Pediatria (São Paulo) 2003;25:128-33.

17. Newburger JW, Takahashi M, Beiser AS, Burns JC, Bastian J, Chung KJ et al. A single intravenous infusion of gamma globulin as compared with four infusions in the treatment of acute Kawasaki syndrome. N Engl J Med 1991;324:1633-9.

18. Tse SM, Silverman ED, McGrindle BW, Yeung RS. Early treatment with intravenous immunoglobulin in patients with Kawasaki disease. J Pediatr 2002;140:450-5.

19. Fong NC, Hui YW, Li CK, Chiu MC. Evaluation of the efficacy of treatment of Kawasaki disease before day 5 of illness. Pediatr Cardiol 2004;25:31-4.

20. Rosenfield EA, Corydon KE, Shulman ST. Kawasaki disease in infants less than one year of age. J Pediatr 1995;126:524-9.

21. Sittiwangkul R, Pongprot $Y$, Thongsongkrit W, Silvilairat S, Phornphutkul $C$ Kawasaki disease in Thai infants compared with older children. Ann Trop Paediatr 2004;24:59-63.

22. Esposito A, Netto Z, Massaroppe B, Lotufo JP. Kawasaki disease simulating sepsis: case report. Pediatria (São Paulo) 2005;27:287-93.

23. Kim DS. Kawasaki disease. Yonsei Med J 2006;47:759-72.

24. Nakamura Y, Yashiro M, Uehara R, Oki I, Watanabe M, Yanagawa H. Epidemiologic features of Kawasaki disease in Japan: results from the nationwide survey in 2005-2006. J Epidemiol 2008;18:167-72.

25. Fischer TK, Holman RC, Yorita KL, Belay ED, Melbye M, Koch A. Kawasaki syndrome in Denmark. Pediatr Infect Dis J 2007;26:411-5.

26. Lima AB, Barros M, Santos M. Description of the social markers in adolescents with rheumatic fever. Rev SOCERJ 2005;18:36-40. 